SCIREA Journal of Clinical Medicine

ISSN: 2706-8870

http://www.scirea.org/journal/CM

January 26, 2021

SCIREA

Volume 6, Issue 1, February 2021

\title{
Imaging Conundrum of Granular Cell Astrocytoma: A Case Report
}

Fan Zhu ${ }^{1, *}$, Sonal Paul', Eric Miller ${ }^{3}$, Chathula Kumarasinghe ${ }^{4}$, Bahaa Mokhtar ${ }^{5}$, Madhumathi Kalavar 6

${ }^{1}$ Department of Radiation Oncology, New York - Presbyterian Brooklyn Methodist Hospital, Brooklyn, New York, USA

${ }^{2}$ Department of Hematology and Oncology, New York - Presbyterian Brooklyn Methodist Hospital, Brooklyn, New York, USA

${ }^{3}$ Department of Radiology, Ohio State University, Columbus, OH, USA

${ }^{4}$ Department of Medicine, New York - Presbyterian Brooklyn Methodist Hospital, Brooklyn, New York, USA

${ }^{5}$ Department of Radiation Oncology, New York - Presbyterian Brooklyn Methodist Hospital, Brooklyn, New York, USA

${ }^{6}$ Department of Hematology and Oncology, New York - Presbyterian Brooklyn Methodist Hospital, Brooklyn, New York, USA

*Corresponding Author:

Department of Radiation Oncology, New York - Presbyterian Brooklyn Methodist Hospital, Brooklyn, New York

Contact Address: 506 6th Street, Department of Radiation Oncology, Brooklyn, NY, 11215

Email: faz9008@nyp.org 
Phone: $718-780-3677$

Fax: 718-780-3637

ORCID: 0000-0002-2063-1630

\begin{abstract}
:
Introduction: Granular cell astrocytoma (GCA) does not have defined radiological criteria/appearance for diagnosis. Here we present a case of GCA that was initially diagnosed as primary CNS lymphoma before pathologic diagnosis was available.

Case Description: We report a 73 year old male patient who presented to our ED with tonicclonic seizures with additional seizures at ED. Initial CT showed no intracranial hemorrhages and MRI brain showed T2 intense lesions in the left inferior basal ganglia, left temporal stem and left amygdala. CT abdomen and pelvis showed thickened rectosigmoid junction, enlarged prostate and no lymphadenopathy in the chest, abdomen or pelvis. MRI brain spectroscopy showed increased choline peak, decrease of NAA peak and no appreciable lactate peak and it also showed that the lesion was homogeneously enhancing with rapid growth rate. Considering all these appearances, a diagnosis of primary CNS lymphoma was considered. Patient then had a left temporal stereotactic craniotomy and microsurgical subtotal resection of the left temporal mass. A gross total resection was not possible due to the location of the lesion. Pathology showed cells with abundant granular cytoplasm, perivascular lymphocytic and histiocytic infiltrates with strong nuclear activity for Olig2 and nonreactivity for CD 163 or MNDA. A final pathological diagnosis of granular cell astrocytoma was made. Patient was treated with $60 \mathrm{~Gy}$ radiation to his post-surgical area with concurrent temozolomide. Patient received 2 cycles of temozolomide, which was stopped due to transaminitis. Patient's mental status and neurological status further declined after the treatment and was placed on hospice.
\end{abstract}

Conclusion: Granular cell astrocytoma can show unusual radiological appearances that can mimic primary CNS lymphoma. Pathological verification of the diagnosis is important. It should be treated as high grade glioma.

Keywords: case report, granular cell astrocytoma, MRI spectroscopy, MRI brain, radiation 


\section{Introduction:}

Granular cell astrocytoma (GCA) is a rare adult infiltrating glioma subtype [Vizcaino 2019]. The presence of perivascular lymphocytic infiltrate may alter the typical radiologic appearance of common astrocytomas [Afonso 2020]. Here we present a case of GCA that was initially diagnosed as primary CNS lymphoma before pathologic diagnosis was available.

A 73-year-old man with no known past medical history presented at the emergency department with confusion witnessed by family, fall, and first tonic-clonic seizure. Patient had two more seizures in the emergency department associated with emesis. He was subsequently intubated for airway protection and admitted to the intensive care unit for management of status epilepticus. CT brain without contrast showed no acute intraparenchymal hemorrhage or acute calvarial fracture. Initial MR imaging done at presentation revealed an expansile, T2-hyperintense lesion with mild diffusion restriction centered in the inferior frontal lobe and the left globus pallidus (Fig.1a). Post-contrast imaging revealed patchy peripheral contrast enhancement with heterogeneous central hypoenhancement (Fig.1c). A smaller, weakly-enhancing satellite nodule was also seen in the lateral left temporal lobe (Fig.1c). The two enhancing abnormalities were linked by a non-enhancing, infiltrating signal changes extending through the left temporal stem (not pictured). Initial presentation favored a glioma though primary CNS lymphoma was also on the differential.

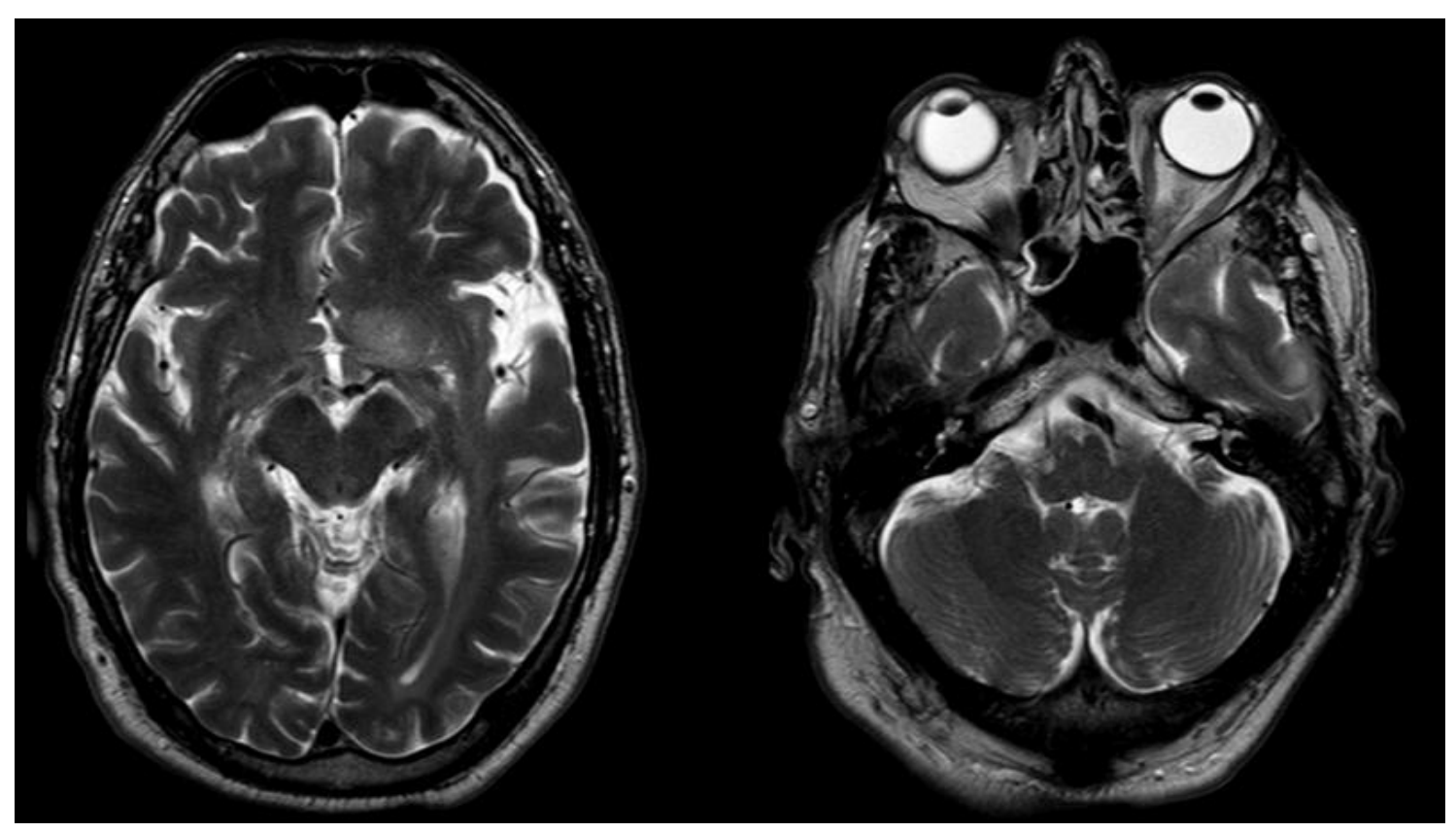

Fig 1a. Initial T2-weighted MRI 


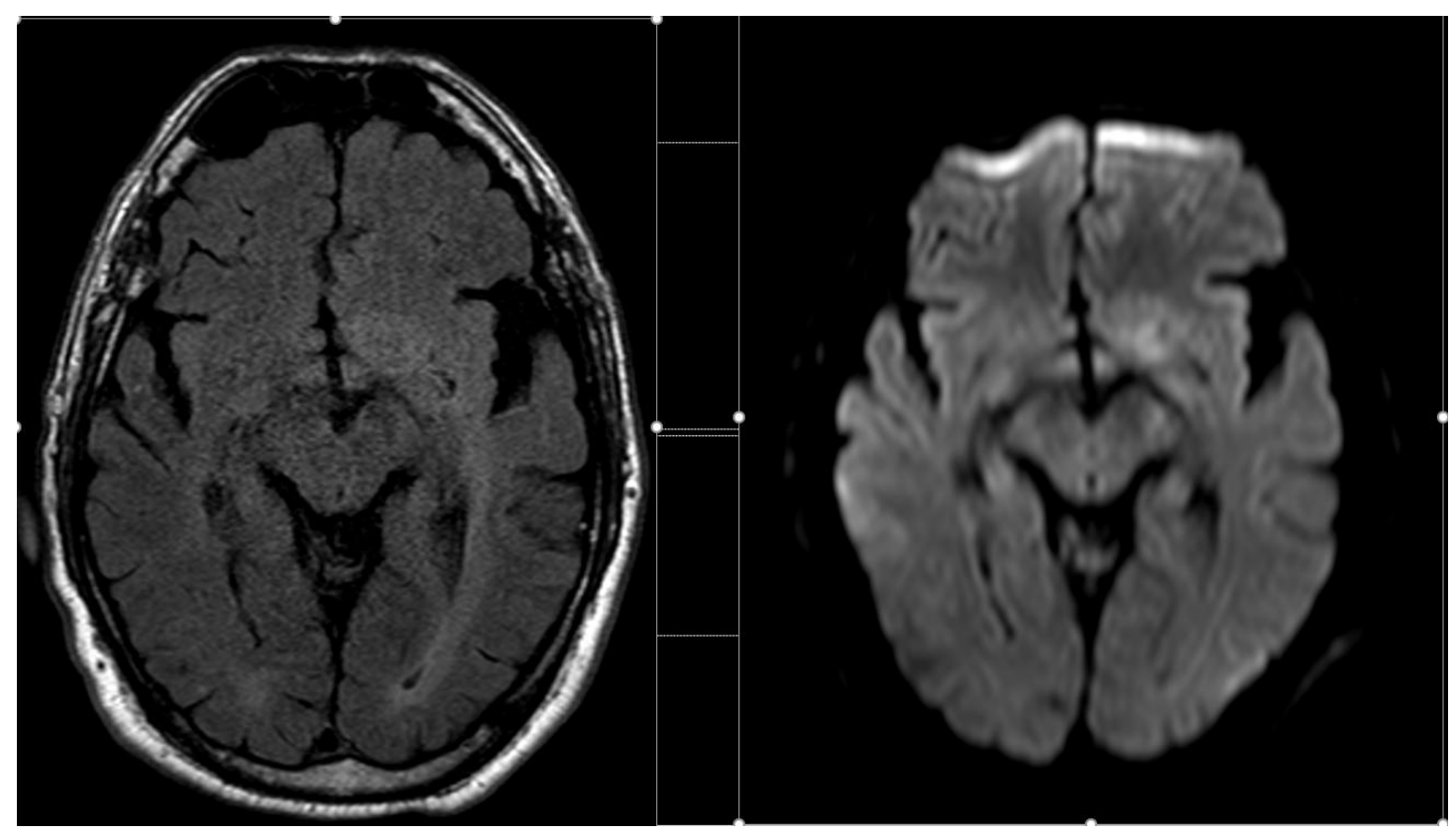

Fig 1b.Initial T2 w/FLAIR (left) and DWI (right) MRI

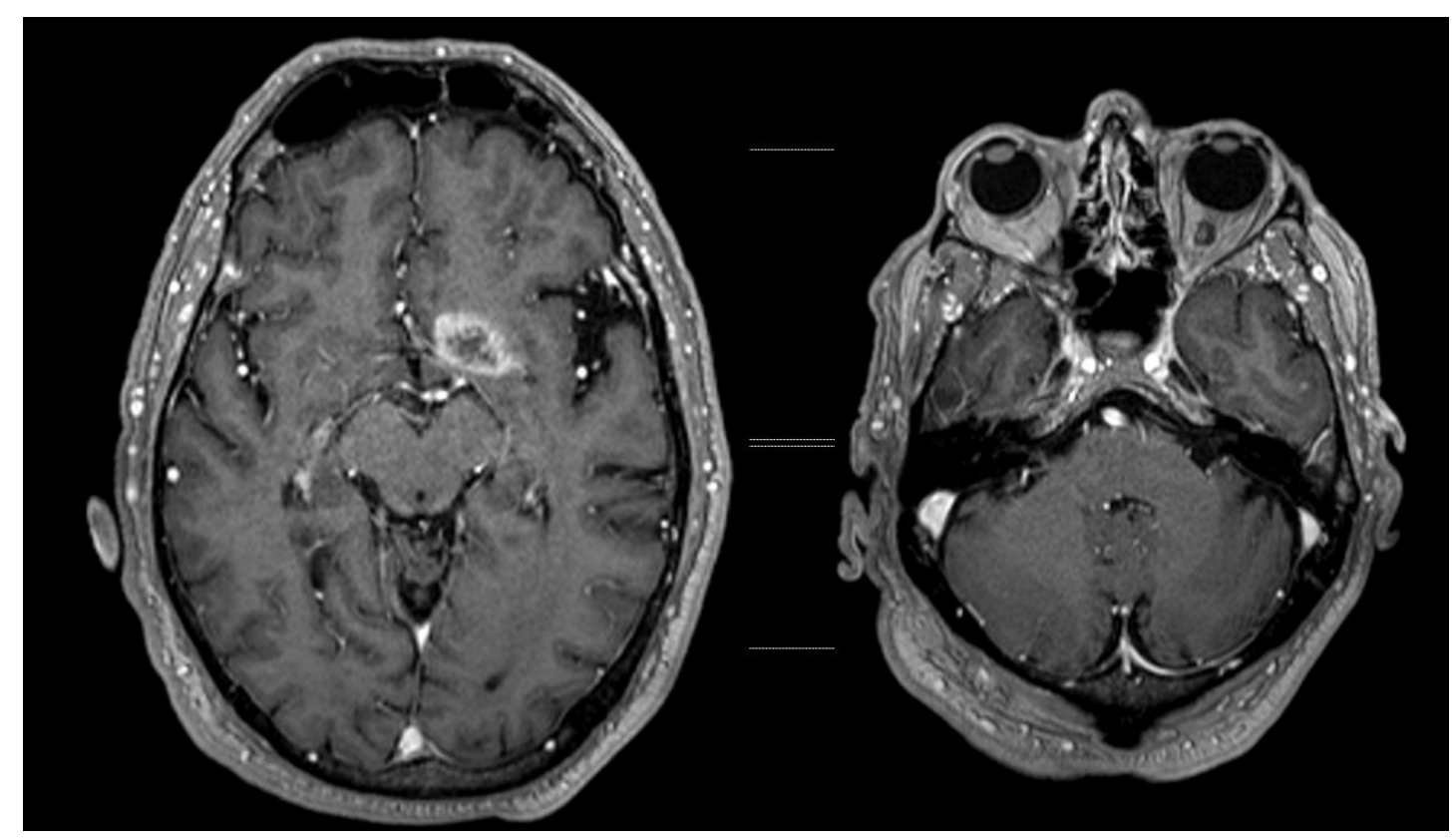

Fig 1c.Initial MRI, T1 with contrast

The patient was extubated on day 3. On day 7 the patient had a lumbar puncture with CSF cytology and flow cytometry negative for malignant cells.

Follow-up imaging on day 11 showed significant short-term interval increase in the size of both the main lesion and the satellite nodule. At this time point the main mass was homogeneously enhancing (Fig.2a). There was also more robust nodular enhancement in the 
temporal satellite nodule (Fig.2a). The amount of surrounding T2 signal changes had also increased in the interim (Fig.2b).

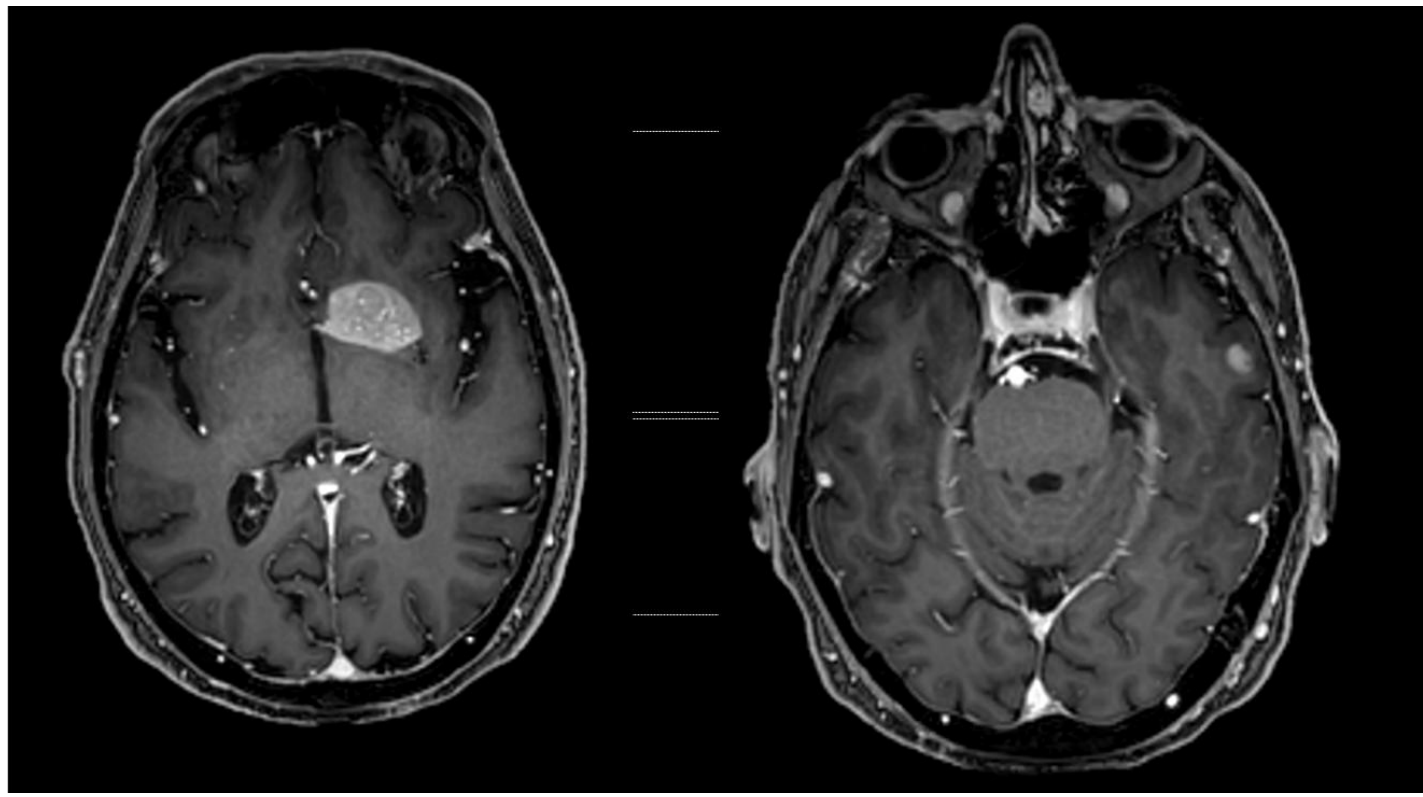

Fig 2a.MRI brain on day 11, T1 with contrast

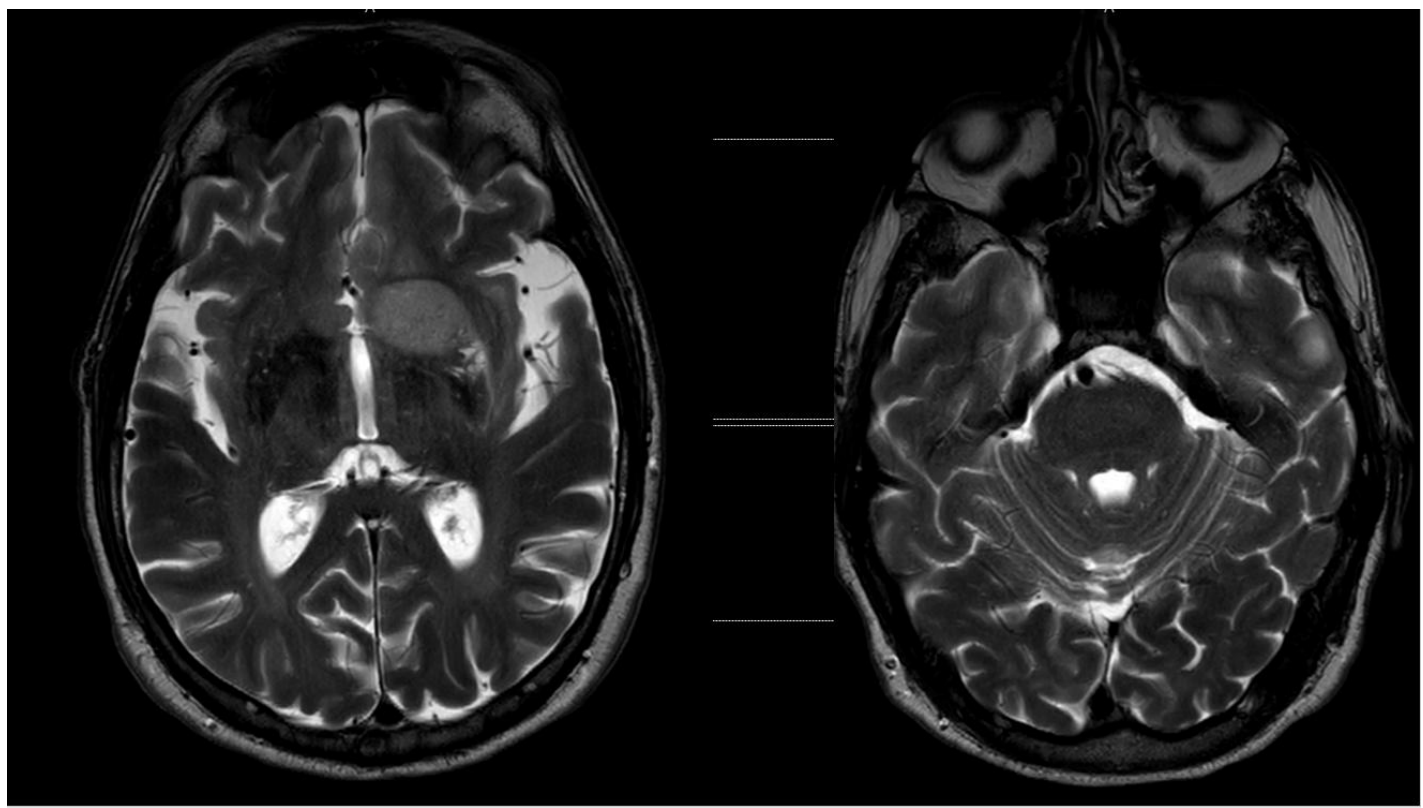

Fig 2b.MRI brain on day 11, T2

MRI brain showed homogeneous enhancement within the mass. MRI diffusion study on day 11 showed restricted diffusion and MR perfusion showed increase in internal cerebral blood volume in the dominant lesion. 
MR Spectroscopy on day 11 demonstrated an elevation of the choline peak, a marker of increased cellular turnover, and marked decrease in the n-acetylaspartate (NAA) peak, a marker of neuronal elements (Fig.2c-2e). The lipid and lactate peaks also remained suppressed, which were surrogates for lesion necrosis (Fig.2c-2e). The pattern of elevated choline and suppressed NAA could be seen in highly cellular lymphomas and high-grade gliomas, though the demonstrated degree of NAA suppression was more often seen in glioblastomas with significant internal necrosis.

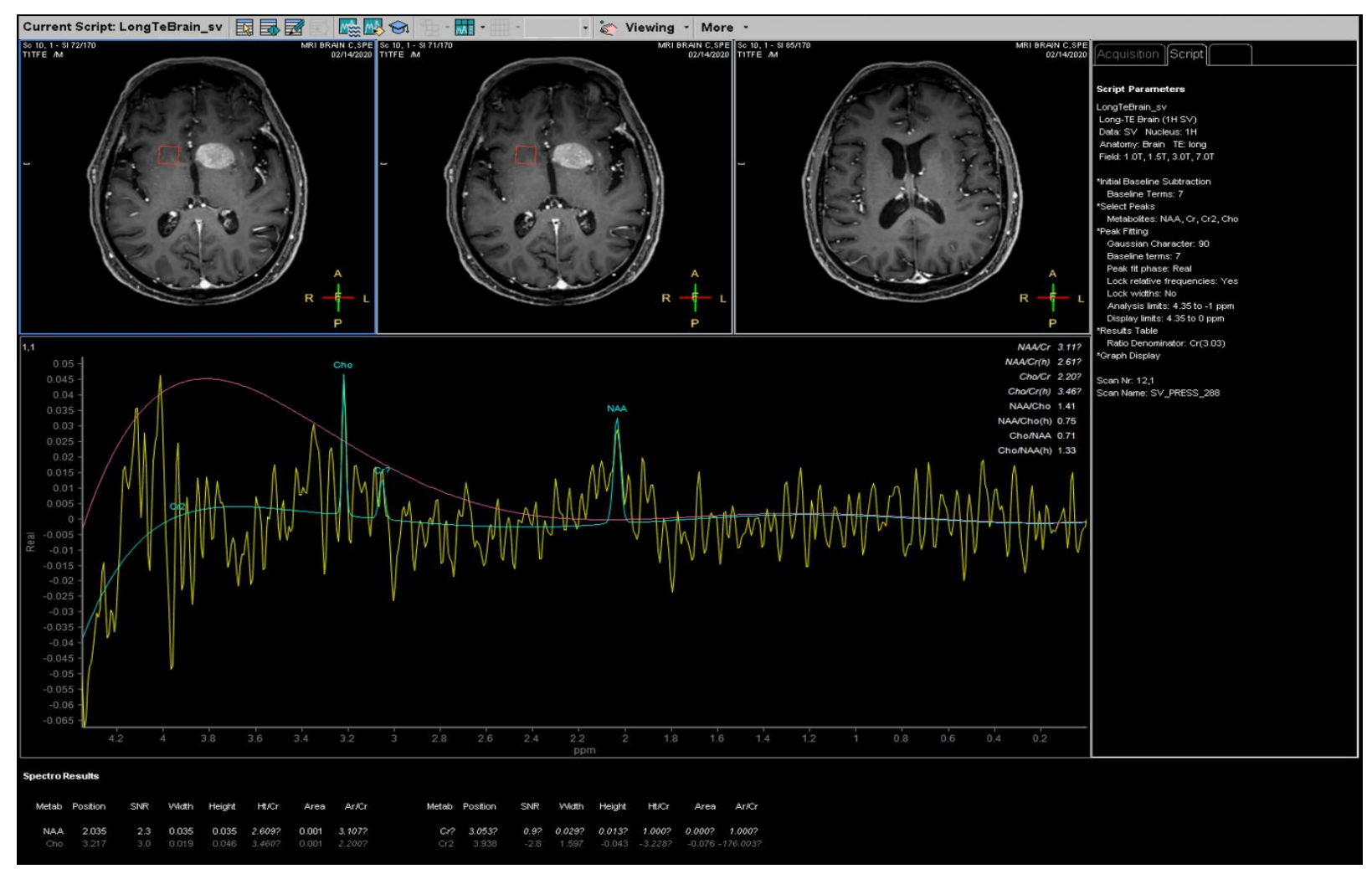

Fig 2c.MRI Spectroscopy on day 11, normal brain tissue 


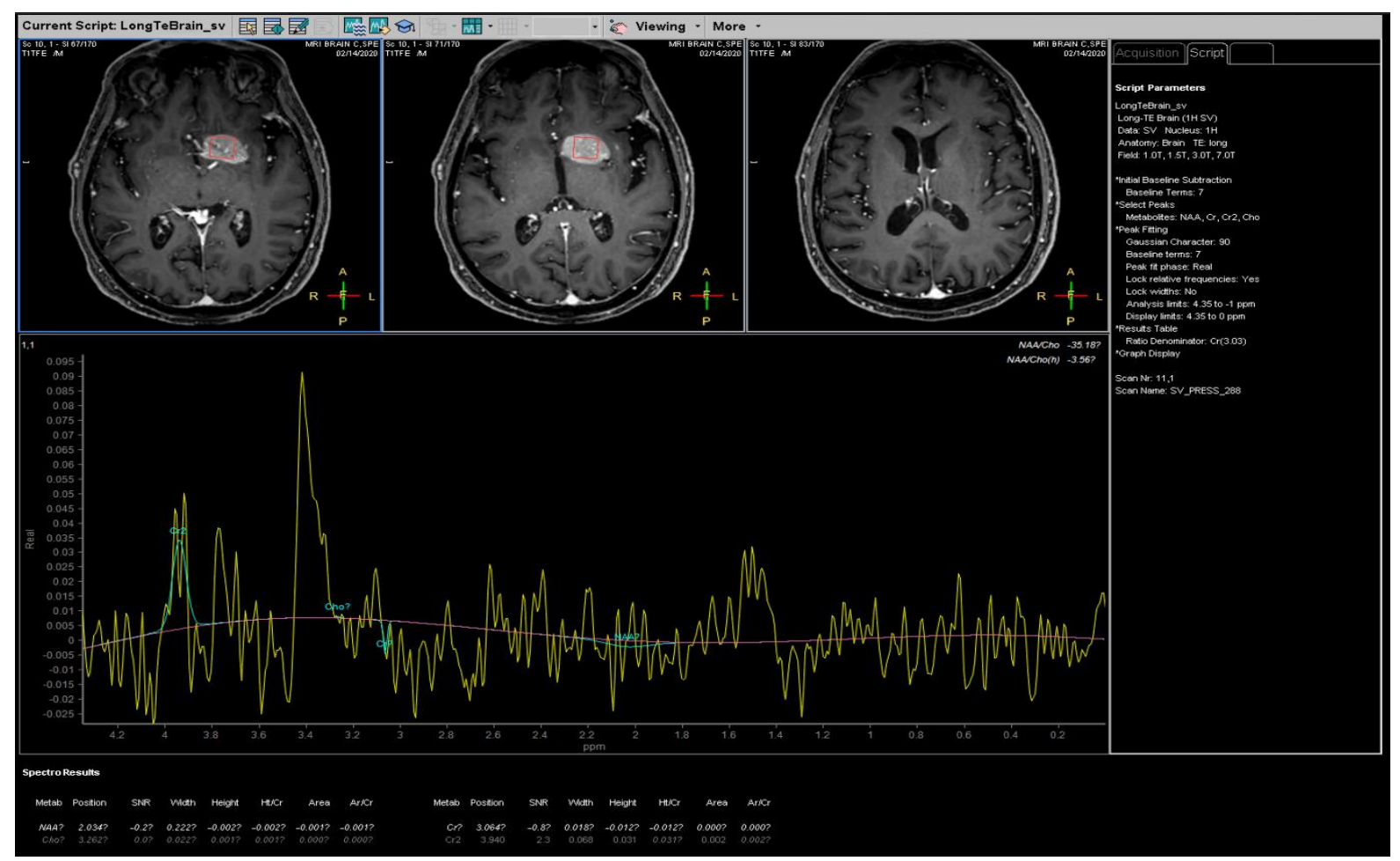

Fig 2d.MRI Spectroscopy on day 11, tumor area

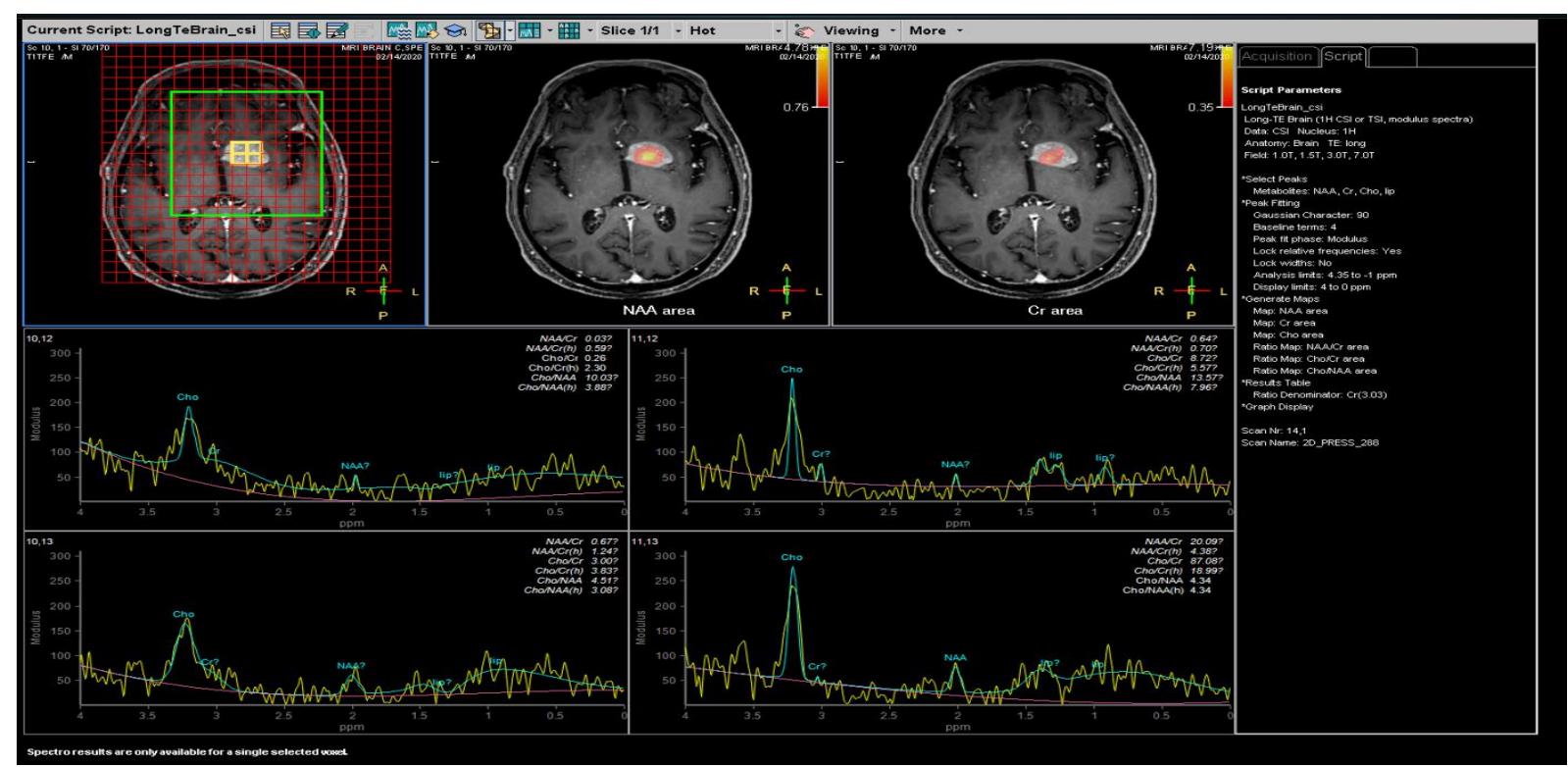

Fig 2e.MRI Spectroscopy on day 11, tumor area

Given rapid increase in size and change in enhancement pattern and the supportive evidence on the MR Spectroscopy, suspicion increased for a primary CNS lymphoma.

On day 16 patient underwent left temporal craniotomy and biopsy. Following biopsy the patient was started on dexamethasone. On Day 19 the patient was discharged from the hospital. Pathology results showed brain parenchyma with marked reactive gliosis and 
perivascular lymphocytic and histiocytic infiltrates with several hypercellular fragments infiltrated by cells with abundant granular cytoplasm. KP-1 (CD68) showed lysosomal staining in the granular cell components, but the majority of the cells was nonreactive for CD 163 or MNDA and showed strong nuclear reactivity for Olig2, supporting an interpretation of an infiltrating glioma. CD3 highlighted foci of lymphocytic cells in a perivascular distribution. Tumor cells were nonreactive for IDH1 R132H with preserved expression of ATRX and scattered nonspecific staining for p53. Ki-67 was up to $6 \%$. Overall features were most consistent with a granular cell astrocytoma. Patient was treated with concurrent chemoradiation.

\section{Radiation:}

Radiation consisted of two phases. Phase 1 included external beam radiation with dose of 4600 cGy in 23 fractions (200cGy/fx) to MRI T2 edema area with two consecutive margins added (PTV_4600). The first margin $(1.5 \mathrm{~cm})$ was to include microscopic disease and the second margin $(0.5 \mathrm{~cm})$ was to include patient set-up uncertainties. We used 6MV photons and volumetric modulated arc therapy (VMAT) technique, which consisted of two counterclockwise arcs and one clockwise arc.

Phase 2 was the boost phase. Phase 2 included external beam radiation with dose of $1400 \mathrm{cGy}$ in 7 fractions $(200 \mathrm{cGy} / \mathrm{fx})$ to T1 MRI contrast-enhanced area in the left temporal lobe with a margin of $1.5 \mathrm{~cm}$ added (PTV_6000) to account for microscopic disease and patient set-up uncertainties. We used 6MV photons and VMAT technique, which consisted of two counterclockwise arcs and one clockwise arc.

He was treated from 3/18/2020 - 4/29/2020, and completed treatment with no unexpected treatment toxicities or delays.

\section{Chemotherapy:}

Concurrent temozolomide was administered with radiation therapy. This was followed by further consolidative chemo. Each cycle of the consolidate chemo consisted of $350 \mathrm{mg}$ temozolomide daily for 5 days every 28 days. Cycle 1 was from 6/8/2020 to 6/12/2020. Cycle 2 was completed on 7/10/20. Patient received 2 cycles of temozolomide, which was stopped due to transaminitis. 
Patient's mental status and neurological status further declined after the treatment and was placed on hospice.

Follow-up MRI brain four months later showed increasing size and significant necrosis of the lesion, now compatible with the biopsy proven high-grade glioma (Fig.3a). The amount of surrounding infiltrating change on the T2-FLAIR sequences has also significantly increased (Fig.3b)

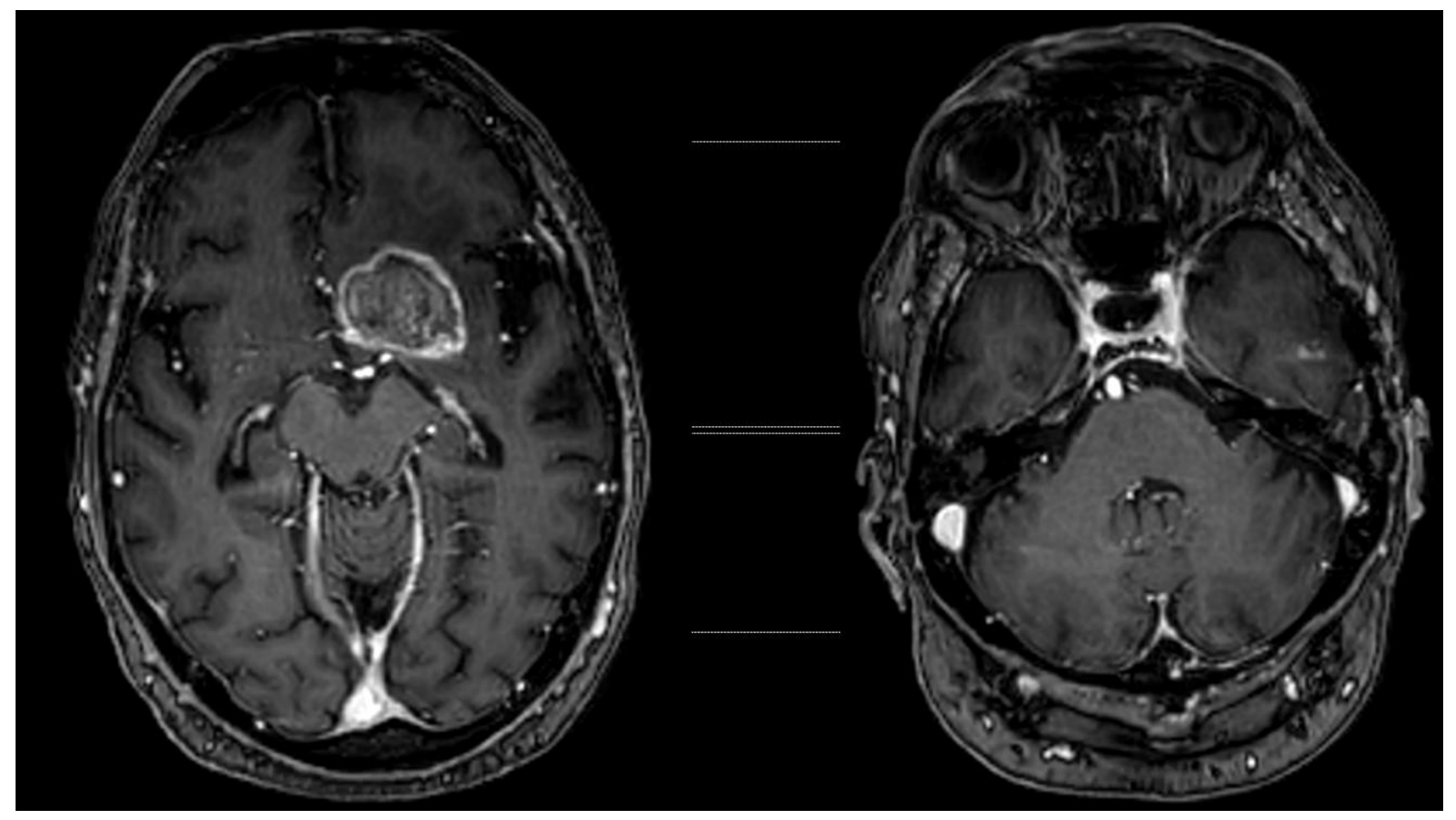

Fig 3a.Follow-up MRI brain four months later, T1 with contrast

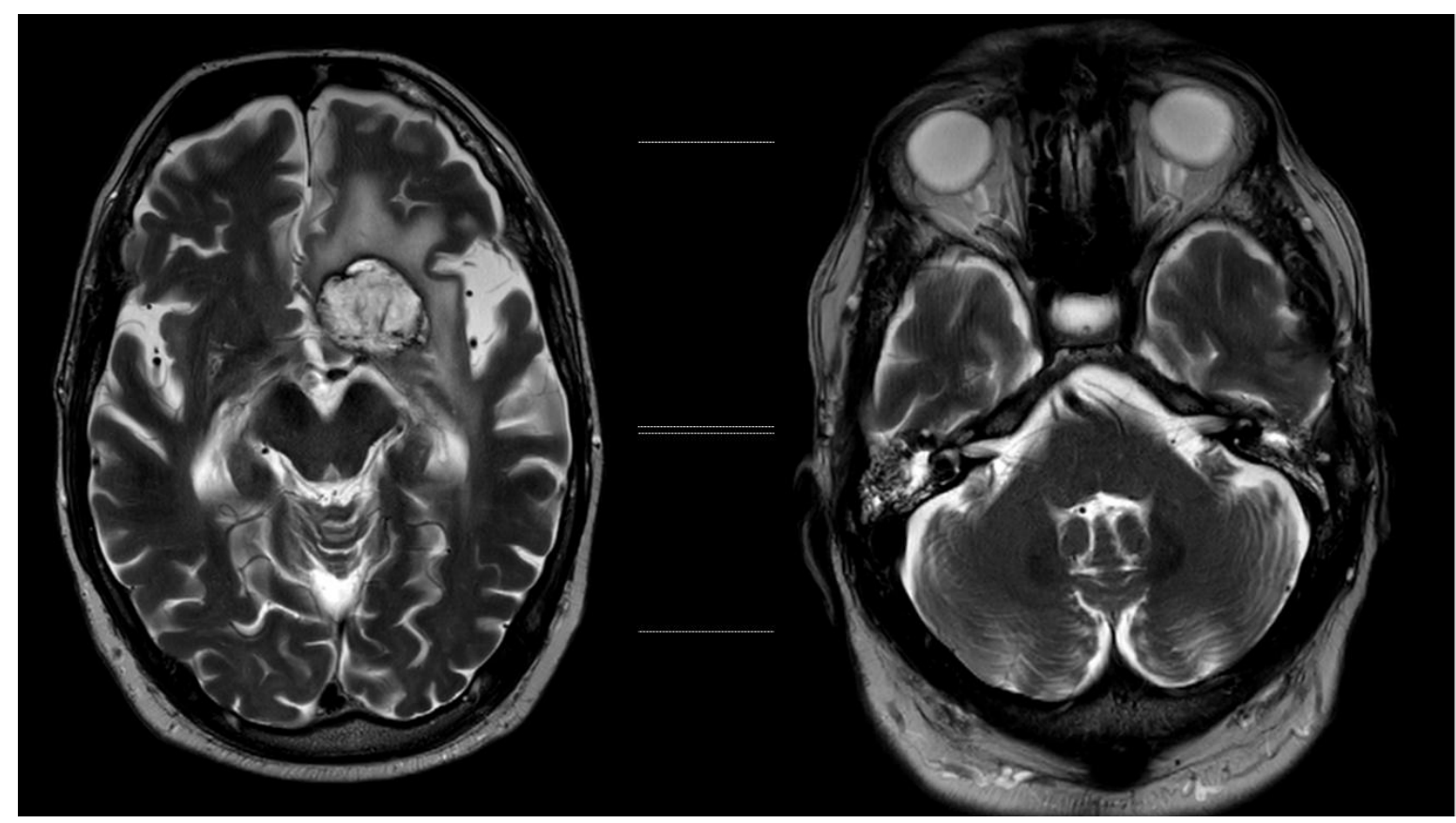

Fig 3b.Follow-up MRI brain four months later, T2 


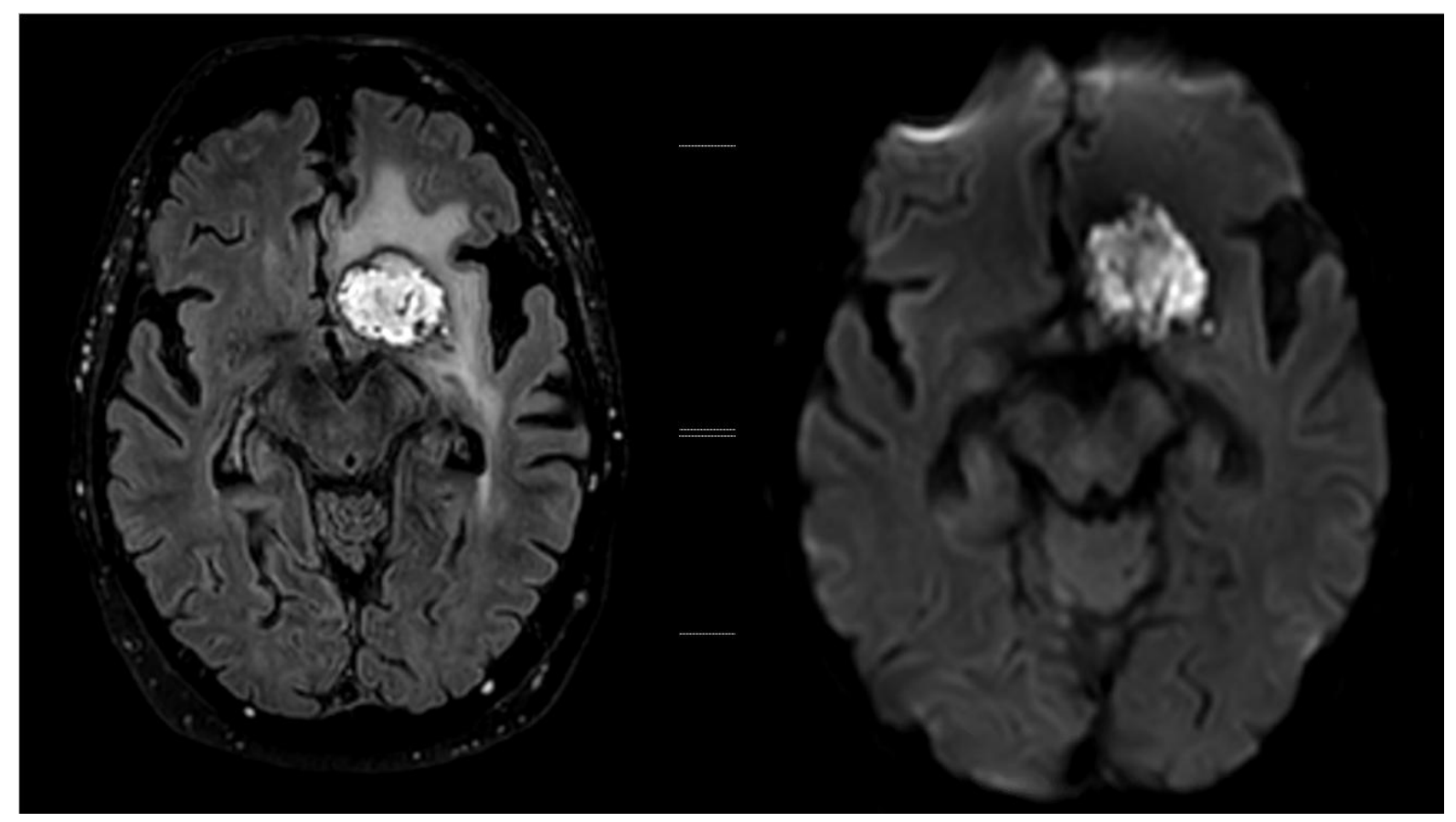

Fig 3c.Follow-up MRI brain four months later, 3D FLAIR Brain view Tra, right: sB1000

Patient had another MRI brain six months later, which showed slightly decreased lesions in the inferior left frontal lobe and inferior left basal ganglia and the central portion showed heterogeneity with peripheral enhancement.

\section{Discussion}

GCA is a variant of IDH-wildtype diffuse glioma with aggressive behavior irrespective of grade and extent of granular cell morphology, and with molecular genetic features corresponding to primary glioblastoma [Vizcaino 2019].

\section{Radiology:}

Radiologic imaging of GCAs showed the same pattern as common astrocytomas in the first published series [Brat 2002]. However, an increase in cases and series reported is evidence of a significant number of GCAs with atypical radiologic features in imaging studies [Brat 2002, Voellger 2015, Imitola 2015 ].

Another case report has shown that GCA can be misdiagnosed as primary CNS lymphoma (PCNSL) [Afonso 2020]. However, in that case report, MRI spectroscopy (MRS) was not done and the chronicity of imaging appearances of brain lesion was not discussed. 
Calli et al states that advances in MRI techniques, including perfusion and diffusion sequences, have allowed more accurate diagnosis of brain gliomas [Calli 2006]. Despite the MRS done pre-operatively, our case still had a diagnosis of PCNSL.

MRS compares the chemical composition of normal brain tissue with abnormal tumor tissue. The basic principle that enables MRS is that the distribution of electrons within an atom causes nuclei in different molecules to experience a slightly different magnetic field. This results in slightly different resonant frequencies, which in turn return a slightly different signal. Each metabolite has its own significance and its own resonant frequency (Table 1).

Metabolites

Resonant frequency (ppm, parts per million)

Lactate

Lipids

$\mathrm{N}$-acetylaspartate (NAA)

choline

Table 1. Metabolite and resonance frequency

Choline is a precursor of acetylcholine $(\mathrm{ACH})$, a component of cell membranes and is a marker of cellular membrane turnover and therefore elevated in neoplasms, demyelination, inflammation and gliosis [Mader 2008]. The synthesis of NAA, the acetylated form of the amino acid, aspartate, occurs in the neuronal mitochondria and therefore, NAA is found in high concentrations in neurons and a marker of neuronal viability [Clark 1998]. It is therefore reduced in any process that destroys neurons, such as high grade tumors, radionecrosis, non-neuronal tumors (e.g. cerebral metastases and primary CNS lymphoma). Lactate is a marker of anaerobic metabolism with no peak seen in normal spectra and is therefore elevated in necrotic areas (e.g. higher grade tumors) and infections (cerebral abscess) [Hesselink]. Lipids are markers of severe tissue damage with liberation of membrane lipids, as is seen in cerebral infarction or cerebral abscesses and is also encountered in cerebral metastases and glioblastoma [Hshimaru 2001]. In our patient, MRS showed an elevation of the choline peak, marked decrease in the NAA peak, and suppressed lipid and lactate peaks. The demonstrated degree of NAA suppression was more often seen in glioblastomas with significant internal necrosis. Given rapid increase in size and change in enhancement pattern and the supportive evidence on the MR Spectroscopy, PCNSL was initially suspected. 


\section{Pathology:}

The most distinctive morphologic feature of GCA is its prominent component of granular cells, which is believed to be intracytoplasmic lysosomes [Shi 2008]. Perivascular lymphocytic cuffing is often noticed [Shi 2008]. GFAP staining can be low $(<30 \%)$ and in some cases can be focal and patchy [Brat 2002]. GCA is positive for CD 68, a 110-kd lysosomal glycoprotein [Liao 2009]. GCA can also be positive for Oligo2 stain. One series of 39 GCA patients showed that Oligo2 was positive in all of the cases examined [Brat 2002]. In our case, the combination of granular cytoplasm, perivascular lymphocytic infiltrates with confirmation of CD3, positive CD 68 and Olig2 point towards a diagnosis of GCA.

\section{Radiology-Pathology correlation:}

The dense perivascular infiltrate of lymphocytes in the GCA can disrupt the blood-brain barrier, allowing leakage of contrast medium and increasing the cellularity of the tumor, restricting water diffusion on DWI [Afonso 2020]. Therefore, GCAs, regardless of the grade, can have an aggressive appearance on conventional and advanced MRI, which, in the absence of central necrosis, may lead to the diagnosis of other nonnecrotic, high-cellularity malignant tumors such as PCNSLs [Afonso 2020]. Cerebral blood volume (CBV) can be used to differentiate PCNSL from high grade glioma. One study has shown that the relative cerebral blood volume (rCBV) in PCNSL is much lower than that in high grade glioma [Liao 2009].

More studies are needed to establish the role of lymphocyte infiltration among the radiologic features of GCAs and their poor prognosis. Recent studies suggest that the balance of lymphocyte subpopulations may influence clinical outcome in patients with glioblastoma [Han 2014, Sayour 2015]. The abscence of IDH mutations in our GCA case confirm the hypothesis that GCA is a variant of IDH wild-type diffuse glioma described by Vizcaino et al [Vizcaino 2019].

\section{Prognosis:}

Due to the aggressive clinical behavior, most GCA patients die within a year [Brat 2002]. One study has shown that among 18 cases with follow-up, 15 recurred after surgery and resulted in death with mean survival of 7.6 months [Brat 2002]. Castellano-Sanchez et al 
found that GCA displayed a higher frequency of loss of heterozygosity at $9 p$ and $10 q$ than the conventional astrocytoma of similar grade, which may contribute to its aggressive clinical behavior [Castellano-Sanchez 2003].

\section{Conclusions}

GCA is a rare and clinically aggressive histologic variant of astrocytomas with poor clinical prognosis. Pathologically, GCAs have classic lysosomal CD 68+ granucles, perivascular lymphocytic infiltrates and Oligo2 positivity. An aggressive radiologic appearance with MRS analysis resembling lymphoma, homogenous contrast enhancement and restriction of diffusion regardless of the grade of malignancy can lead to a wrong diagnosis. Lymphocytic perivascular infiltrates may explain these atypical features.

\section{References:}

[1] Vizcaino M.A., Palsgrove D.N., Yuan M., et. al.: Granular cell astrocytoma: an aggressive IDH-wildtype diffuse glioma with molecular genetic features of primary glioblastoma. Brain Pathol 2019; 29: pp. 193-204

[2] Case Reports , World Neurosurg. 2020 Feb;134:164-169. doi:10.1016/j.wneu.2019.10.169. Epub 2019 Nov 4. Imaging and Radiologic-Pathologic Correlation in Granular Cell Astrocytomas: Report of 2 Cases Antonio Montalvo Afonso, Juan Vicente Darriba Alles, Ángela Moreno Gutiérrez, Lain Hermes González Quarante , Roberto García Leal, Juan Adan Guzmán de Villoria Lebiedziejewski , Emma Sola Vendrell

[3] Brat D.J., Scheithauer B.W., Medina-Flores R., Rosenblum M.K., Burger P.C.: Infiltrative astrocytomas with granular cell features (granular cell astrocytomas): a study of histopathologic features, grading, and outcome. Am J Surg Pathol 2002; 26: pp. 750757

[4] Voellger B., Tapia-Perez J.H., Rupa R., et. al.: Survival in granular cell astrocytomas. J Neurol Surg A Cent Eur Neurosurg 2015; 76: pp. 30-38. 
[5] Imitola J., Wagoner J., Khurana J.S.: Rapid dissemination of granular cell astrocytoma arising from periventricular stem cell regions in chronic multiple sclerosis. J Neurooncol 2015; 124: pp. 147-149.

[6] Calli C., Kitis O., Yunten N., Yurtseven T., Islekel S., Akalin T.: Perfusion and diffusion MR imaging in enhancing malignant cerebral tumors. Eur J Radiol 2006; 58: pp. 394403.

[7] Mader I, Rauer S, Gall P, Klose U. (1)H MR spectroscopy of inflammation, infection and ischemia of the brain. (2008) European journal of radiology. 67 (2): 250-7. doi:10.1016/j.ejrad.2008.02.033

[8] Clark JB. N-acetyl aspartate: a marker for neuronal loss or mitochondrial dysfunction. (1998) Developmental neuroscience. 20 (4-5): 271-6. doi:10.1159/000017321 - Pubmed

[9] Hesselink, JR. Fundamentals of MR Spectroscopy. Available online: http://spinwarp.ucsd.edu/NeuroWeb/Text/mrs-TXT.htm

[10] Ishimaru H, Morikawa M, Iwanaga S et-al. Differentiation between high-grade glioma and metastatic brain tumor using single-voxel proton MR spectroscopy. Eur Radiol. 2001;11 (9): 1784-91.

[11] Shi Y, Morgenstern N. Granular cell astrocytoma. Arch Pathol Lab Med. 2008 Dec;132(12):1946-50. doi: 10.1043/1543-2165-132.12.1946. PMID: 19061297.

[12] Brat DJ, Scheithauer BW, Medina-Flores R, Rosenblum MK, Burger PC. Infiltrative astrocytomas with granular cell features (granular cell astrocytomas): a study of histopathologic features, grading, and outcome. Am J Surg Pathol. 2002; 26:750-757.

[13] Liao W., Liu Y., Wang X., et. al.: Differentiation of primary central nervous system lymphoma and high-grade glioma with dynamic susceptibility contrast-enhanced perfusion magnetic resonance imaging. Acta Radiol 2009; 50: pp. 217-225.

[14] Han S., Zhang C., Li Q., et. al.: Tumour-infiltrating CD4(+) and CD8(+) lymphocytes as predictors of clinical outcome in glioma. Br J Cancer 2014; 110: pp. 2560-2568.

[15] Sayour E.J., McLendon P., McLendon R., et. al.: Increased proportion of FoxP3+ regulatory $\mathrm{T}$ cells in tumor infiltrating lymphocytes is associated with tumor recurrence and reduced survival in patients with glioblastoma. Cancer Immunol Immunother 2015; 64: pp. 419-427. 
[16] Brat DJ, Scheithauer BW, Medina-Flores R, Rosenblum MK, Burger PC. Infiltrative astrocytomas with granular cell features (granular cell astrocytomas): a study of histopathologic features, grading, and outcome. Am J Surg Pathol. 2002; 26:750-757.

[17] Castellano-Sanchez AA, Ohgaki H, Yokoo H, et al. Granular cell astrocytomas show a high frequency of allelic loss but are not a genetically defined subset. Brain Pathol. 2003;13:185-194. 